
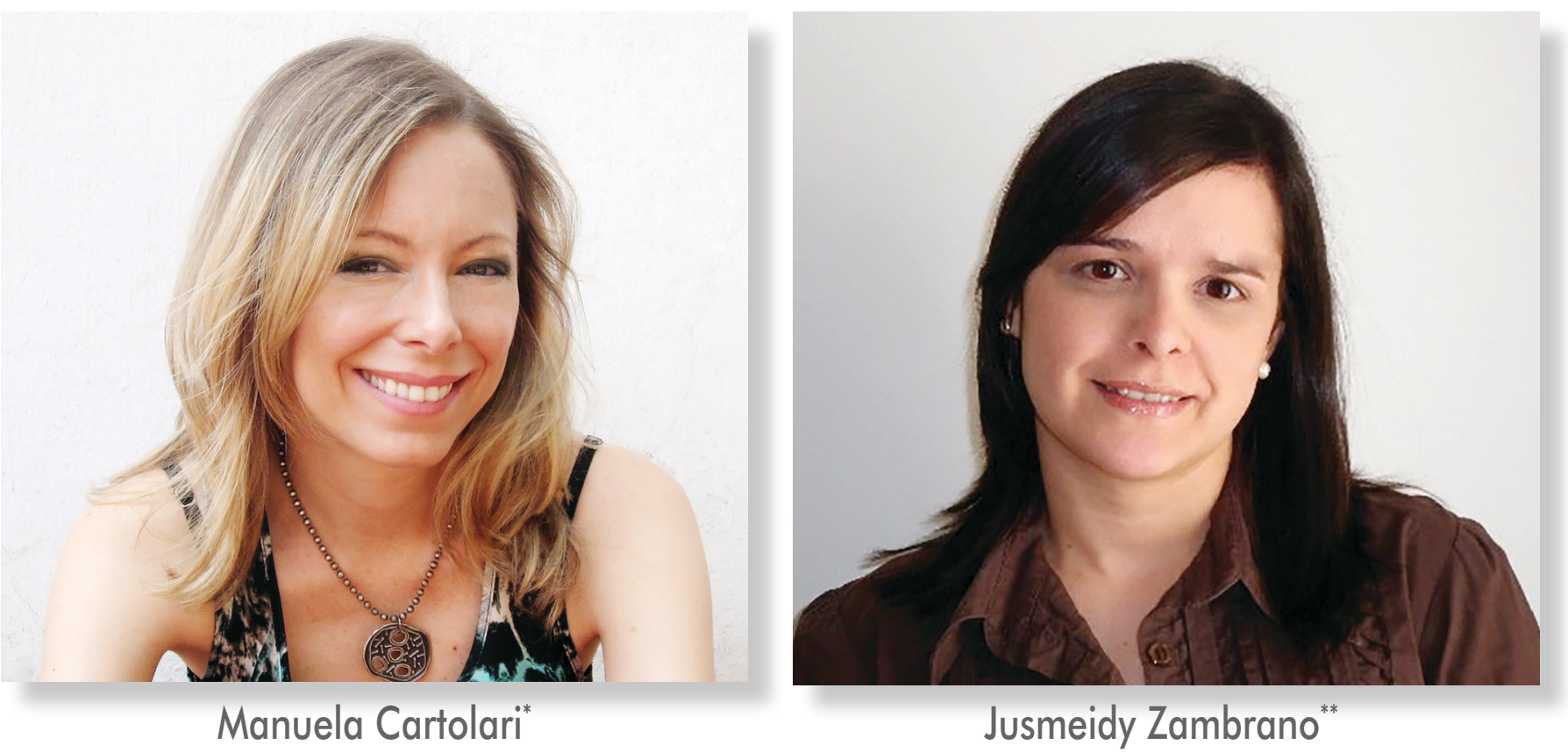

\title{
Reseña del libro Escribir, leer y aprender en la universidad: una introducción a la alfabetización académica ${ }^{1}$
}

\author{
Si tiene remedio, ¿por qué te quejas? \\ Si no tiene remedio, ¿por qué te quejas? \\ (Proverbio oriental)
}

\section{Palabras claves}

lectura; escritura; alfabetización académica; universidad

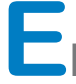

libro que reseñamos, "Escribir, leer y aprender en la universidad: una introducción a la alfabetización académica" (2005) ${ }^{1}$, escrito por la reconocida investigadora Dra. Paula Carlino², se hace eco del acápite de esta reseña al buscar desconstruir nuestras habituales quejas como docentes o actores institucionales sobre lo poco que leen y lo mal que comprenden y escriben los estudiantes en la universidad. Si bien la primera edición de este libro celebrará pronto su décimo aniversario, la vigencia y relevancia de su mensaje no se han visto reducidas por el correr de los años.

\footnotetext{
"Manuela Cartolari: Doctora en Educación por la Universidad Nacional de Lanús y la Universidad Nacional de Tres de Febrero (Argentina). Licenciada en Psicología por la Universidad de Buenos Aires. Becaria Post-doctoral del Consejo Nacional de Investigaciones Científicas y Técnicas (CONICET) de Argentina. Integrante del GICEOLEM (Grupo para la Inclusión y Calidad Educativas a través de Ocuparnos de la Lectura y la Escritura en todas las Materias).https://sites.google.com/site/ giceolem2010/ Para contactar a la autora: manucartolari@yahoo.com

"Jusmeidy Zambrano: Doctoranda en Ciencias de la Educación de la Universidad Nacional de La Plata. Profesora de la Universidad Nacional Experimental del Táchira (Venezuela). Investigadora "A" del Programa de Estímulo a la Innovación e Investigación (Venezuela). Integrante del GICEOLEM (Grupo para la Inclusión y Calidad Educativas a través de Ocuparnos de la Lectura y la Escritura en todas las Materias). https://sites.google.com/site/giceolem2010/ Para contactar a la autora: jzambran@unet.edu.ve
} 
Al parecer, no ha sido ni sigue siendo fácil encontrar caminos viables para emprender y difundir acciones sistemáticas que, como muestra esta obra en forma precisa y fundamentada, se hagan cargo de que el "problema" de la lectura y la escritura en el nivel superior sí tiene remedio y es "un asunto de todos" (Carlino \& Martínez, 2009).

Ocuparse de la lectura y escritura en la universidad significaría, según la autora, suplantar las quejas habituales enfocadas en los déficits de los alumnos por investigaciones e iniciativas de transferencia a la práctica que logren que leer y escribir, tal como pone de manifiesto el libro reseñado, se utilicen como herramientas de aprendizaje y, a la vez, se conviertan en objetos de enseñanza. Ello consiste en una problemática cuya propia resistencia al cambio nos obliga a repensar cómo hemos interpretado -o incomprendido- el desafío que nos plantea. Un dato alentador, no obstante, aparece de la mano de diversos proyectos que buscan, tal como se informa en este mismo número de Cuaderno de Pedagogía Universitaria, incorporar la lectura y/o escritura en el marco institucional del Programa de Alfabetización Académica que la Pontificia Universidad Católica Madre y Maestra (PUCMM) ha iniciado desde hace ya cuatro años.

El libro de Paula Carlino resulta, a todas luces, una obra seminal en América Latina. En efecto, ha sido distinguido como Mejor Libro de Educación -Obra didáctica- de edición 2005 por la Feria del Libro de la Ciudad de Buenos Aires (Argentina), y cuenta ya con siete reimpresiones y más de 14.000 copias vendidas. Además, es la publicación más citada entre la prolífica producción escrita de la autora, tanto en nuestra región como en el contexto internacional.

Entonces, ¿qué hace todavía vigente y novedoso este libro? Desde nuestro punto de vista, se trata, en primer lugar, de la actualidad de las preguntas que formula desde su página inicial: "¿por qué ese desencuentro entre lo que los profesores esperamos [sobre cómo leen, escriben y aprenden nuestros alumnos] y lo que logran los estudiantes en el nivel superior?" y, sobre todo, "¿qué podemos hacer los docentes para disminuir esta brecha en parte inevitable?" (p. 9).

En segundo término, aunque sin por eso merecer menor importancia, la calidad de la obra reseñada es resultado de las razones que motivaron a la autora a plantearse tales interrogantes y de cómo buscó responderlos. En ese sentido, los antecedentes de este texto pueden rastrearse en el trabajo de capacitación a docentes en servicio que Carlino realizó, en conjunto con Myriam Nemirovsky y Denise Santana, en la Red de Formación Permanente del Profesorado del Ministerio de Educación y Ciencia de España.
Tal como ha sostenido la autora en varias de sus conferencias, fue allí que aprendió que la enseñanza sólo puede transformarse a partir de la investigación sobre la propia práctica, investigación que, a su vez, ha de nutrirse del diálogo con otros profesores y con otras investigaciones provenientes de las múltiples disciplinas que dialogan con la educación (psicología del desarrollo y del aprendizaje, psicología educacional, lingüística, didáctica general y didácticas específicas, antropología educativa, etcétera).

En forma consecuente con este legado, y ya habiendo regresado a Buenos Aires, Paula Carlino puso en práctica estos principios en la cátedra de Teorías del Aprendizaje de la Universidad Nacional de San Martín, de la cual estuvo a cargo entre 1997 y 2002. El libro "Escribir, leer y aprender en la universidad: una introducción a la alfabetización académica" es fruto de su trabajo de investigación-acción durante los cinco años que, como docente de esta asignatura, se propuso sostener los principios constructivistas sobre la lectura y la escritura y el aprendizaje en que se había formado.

De esta manera, la autora, en reiterados ciclos de investigación-acción (Altrichter, Kemmis, McTaggart, \& Zuber-Skerrit, 2002), diseñó, puso a prueba y analizó los resultados de diferentes situaciones de lectura y escritura con un doble propósito. En primer lugar, para favorecer la función epistémica de estas prácticas (Langer \& Applebee, 1987; Wells, 1990a, 1990b), en tanto ciertas tareas que involucran leer y escribir, en determinadas condiciones didácticas y mediante el acompañamiento docente, permiten que los estudiantes desplieguen una mayor actividad cognitiva sobre los contenidos que el sólo escuchar lo que el profesor expone.

Así, la lectura y la escritura pueden ayudar a que los alumnos reelaboren y se apropien de los conocimientos, evitando a la vez que busquen tan sólo memorizarlos para el día de la evaluación. Paralelamente, un segundo propósito orientó las situaciones de lectura y escritura que la autora presenta: convertir estas prácticas en objetos de enseñanza al interior de su materia. Ello implica, según señala Carlino, introducir y andamiar a los alumnos en los modos de leer y escribir propios del campo de conocimientos en el que se adentran, y también a la cultura escrita universitaria a la que han llegado.

En este último punto, el libro reseñado destaca en forma tajante cómo se diferencian los textos que suelen manejar los estudiantes en el secundario de los "textos académicos" que se utilizan en la universidad (pp. 8487). Es en esta clave que debe entenderse el concepto de "alfabetización académica" que se ha hecho tan conocido en Latinoamérica a partir de su obra. 


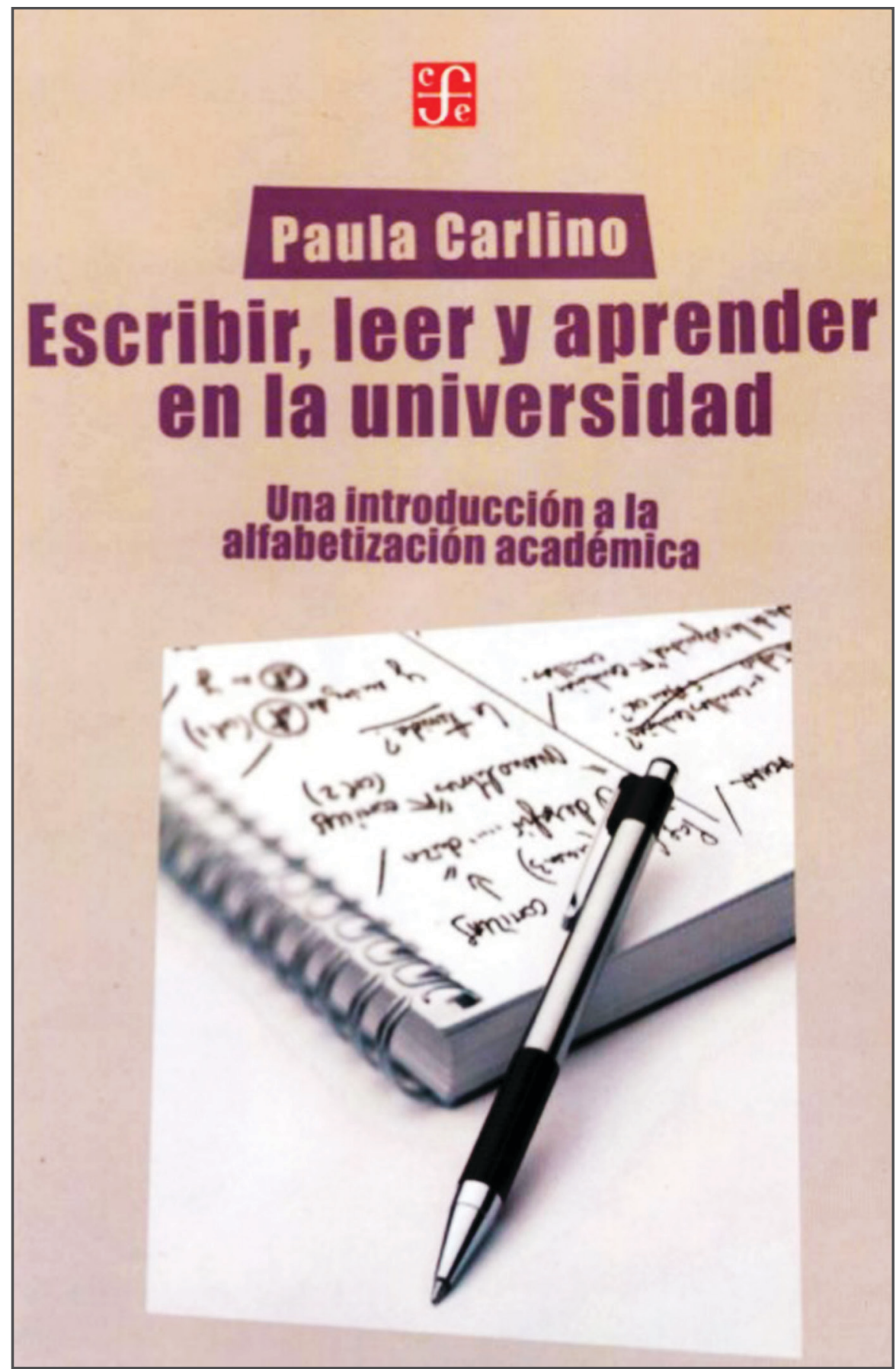

Esta noción -a diferencia de cómo se la ha interpretado algunas veces- no propone un nuevo "contenido", ni sugiere tampoco la necesidad de adicionar algún "espacio curricular" extra para "alfabetizar académicamente" a los alumnos que ingresan al nivel superior.

Por el contrario, la autora resalta que la "alfabetización académica" implica "integrar la producción y el análisis de textos en la enseñanza de todas las cátedras porque leer y escribir forman parte del quehacer profesional/académico de los graduados que esperamos formar" ( $p .15)$. Como argumenta Carlino, este saber hacer con los textos, propio de cada cultura disciplinar, no puede darse como ya sabido por el solo hecho de que un estudiante haya finalizado la educación secundaria, ni tampoco puede ser el resultado esperado de cursos remediales incorporados al comienzo de los planes de estudio de las carreras superiores.

El mensaje sustancial del libro es claro: cada docente necesita reflexionar, explorar, poner a prueba y analizar cuáles son las formas más apropiadas de enseñar a leer y escribir los textos que se manejan en su cátedra.

En forma reiterada se advierte que, para que el esfuerzo que ello significa no sea inconducente, la acción del profesor precisa nutrirse y fundamentarse en distintos aportes teóricos y empíricos que ayuden a hacer coherentes sus propuestas de aula con los objetivos pedagógicos que persigue.

Las anteriores premisas son reflejo cabal de la organización de esta obra, que cuenta con una introducción y cuatro capítulos, además de un índice y las correspondientes referencias bibliográficas. En efecto, Carlino comienza explicitando los presupuestos teóricos básicos que atravesarán las páginas consecutivas (introducción) para luego describir y analizar la implementación de las distintas actividades didácticas y de su propia práctica docente puestas a prueba (tres primeros capítulos).

Por último, a la luz de las actividades y análisis que ha venido exponiendo, el cuarto capítulo reúne y detalla el decálogo de principios teóricos que las atraviesan en su conjunto. Ello tiene como ventaja permitir al lector resignificar, bidireccionalmente, cómo la teoría puede y precisa fundamentar la práctica de los profesores universitarios y, a la vez, cómo los problemas a los que nos enfrenta la práctica pedagógica pueden hallar respuesta recurriendo una y otra vez a nuevos conocimientos y teoría. 
En cuanto a los contenidos que el lector encontrará en cada capítulo, el primero enfoca la escritura en el nivel superior. Al respecto, describe y analiza cuatro situaciones de escritura que la autora experimentó en su materia: "elaboración rotativa de síntesis de clase", "tutorías para escritos grupales", "preparación del examen" y "respuesta escrita a preguntas sobre la bibliografía". Todas las actividades abonan a escribir con conciencia retórica; en cada una de ellas, se ponen en relación los problemas del contenido con los propósitos de la escritura, su audiencia y el género. De esta forma, las acciones propuestas pretenden desarrollar la potencialidad epistémica de la producción textual mediante "situaciones deacompañamientoyorientación a los estudiantes cuando se enfrentan a la tarea de escribir en una materia que aún no dominan" (p.50).

En las últimas páginas de este capítulo, se explica por qué muchos docentes no se ocupan de la escritura de los alumnos y se reflexiona sobre cuán desafiante es para las instituciones hacerse cargo de la lectura y escritura mediante resaltar la experiencia de la propia autora, cuyo trabajo individual no logró trascender, al momento de su desarrollo, el espacio de sus clases.

El segundo capítulo, en cambio, está centrado específicamente en la lectura. En su primera parte, describe y analiza dos tareas: "leer con ayuda de guías" y "resumir para uno mismo". Ambas están destinadas a promover a que los alumnos comprendan la bibliografía a través de jerarquizar lo leído en función de los objetivos de la materia.

Es de destacar que estas actividades, además de orientar el trabajo interpretativo de la bibliografía, también solicitan la reflexión de los estudiantes acerca de los desafíos que la lectura y las tareas requeridas les presentan, de modo que pueda discutirse en clase por qué surgen y cómo superarlos. En la segunda parte de este capítulo, Carlino especifica las marcadas diferencias entre las exigencias de lectura y los tipos de texto que se utilizan en la secundaria y en la universidad, cuestionando así las posturas que responsabilizan al nivel medio de los "déficits de comprensión lectora" que encuentran en los alumnos.

En el tercer capítulo, se proponen y analizan distintas situaciones de evaluación basadas en articular la lectura con la escritura. En primer lugar, se argumenta sobre las funciones que estas instancias pueden cumplir en el aprendizaje, enfatizando la idea de que "la evaluación no es sólo el eslabón final del proceso educativo" (p.106).

La autora plantea un contraste entre las prácticas evaluativas escritas frecuentes y aquellas deseables, y sintetiza en tres palabras lo que significaría una "buena práctica" de evaluación: que sea "válida, explícita y educativa" (p. 113). Seguido a ello, se describen dos situaciones de esta índole, cuyo objetivo es convertir la experiencia del examen en una oportunidad para aprender contenidos en forma entramada con el aprendizaje de la lectura y la escritura académicas: "reescribir el examen" y "leer y escribir para presentar una ponencia oral".

El cuarto y último capítulo, como ya adelantamos, postula y explica diez principios generales que la autora desarrolla a partir de sintetizar y compatibilizar diferentes aportes teóricos sobre el aprendizaje, la enseñanza reflexiva, la investigación pedagógica y la investigación acción como proceso iterativo y espiralado (Altrichter et al., 2002).

Al leer estos principios, se tiene una sensación parecida a la que de seguro todo niño experimenta la primera vez que ve una radiografía y logra observar cómo son por dentro las articulaciones y la estructura ósea que dan movilidad y sostén al cuerpo. De la misma manera, los principios expuestos en este capítulo hacen visible la lógica interna de esta obra y ponen al descubierto el armazón teórico que articula y sostiene las actividades y reflexiones descritas en los capítulos anteriores.

Entre las virtudes del libro reseñado, cabe resaltar la claridad y sencillez de la prosa utilizada para abordar temas que son multidimensionales y complejos, sin que ello reste en nada a la rigurosidad y profundidad con que se analiza cada problemática. Se trata de una obra destinada a un público amplio y diverso, por lo cual interesará tanto a quienes recién se adentran en el tema, como a los lectores más avanzados. Acorde con esta intencionalidad, cada capítulo puede leerse en forma independiente de los demás.

Asimismo, se incorporan a su interior diferentes recuadros, secciones y apartados que permiten detenerse a profundizar en conceptos claves de la teoría o de las situaciones de lectura y escritura propuestas o, en caso contrario, facilitan al lector apresurado saltearse cierta información sin por eso perder el hilo explicativo principal.

Sin embargo, a nuestro juicio, y aunque tales recuadros y apartados son algunos de los elementos más provechosos del libro, el objetivo de facilitar la lectura que tienen podría presentar un efecto adverso si el lector decide saltearlos sin más, ya que con ello se correría el riesgo de acabar realizando una interpretación un tanto superficial y desvirtuada del contenido de este trabajo.

En efecto, la importancia de estos recuadros y apartados reside en que es allí donde la autora se explaya con mayor profundidad sobre: 
a. la fundamentación teórica de cada situación de lectura y escritura puesta a prueba, como también aquella de sus propias prácticas docentes al implementarlas, $y$

b. la descripción y análisis de los géneros escritos que utiliza en sus clases y de las prácticas de estudio que propone realizar a sus alumnos.

De todos modos, sobra mencionar, como seguramente el lector de esta reseña ya ha anticipado, que en este libro no encontrará un "manual" de estrategias o técnicas que simplifique sin más los desafíos de incluir la lectura y la escritura en sus prácticas de enseñanza.

Tal como se va mostrando al describir las actividades de comprensión y producción de textos que se implementan, los resultados obtenidos no son siempre exitosos. En ello radica, justamente, otra de las virtudes que es preciso señalar: Paula Carlino, a lo largo de esta obra, nos brinda un recuento detallado de las dificultades que enfrentan los estudiantes al realizar las tareas que les solicita, como también de los desafíos que ella como docente e investigadora va experimentando.

Acto seguido, nos muestra cómo utiliza la teoría para repensar y ajustar su enseñanza en aras de solucionar los obstáculos que surgen, a los fines de no renunciar a su propósito inicial de enseñar a leer y a escribir para que los alumnos aprendan más y mejor.

En definitiva, y al igual que lo haría un posadero o anfitrión atento a familiarizar al huésped recién llegado con la cultura y las costumbres comunicativas locales, el espíritu que atraviesa esta obra puede resumirse en la actitud de bienvenida y ayuda al estudiante recién llegado a una determinada cultura escrita universitaria y disciplinar, para que comience a sentirse un integrante de la misma y acreciente su participación y autonomía en el nuevo contexto discursivo.

Pero dicha bienvenida no sólo está destinada a los alumnos: este libro es también, en sí mismo, una acogida que presta apoyo de valor a los docentes que quieran ocuparse de la lectura y escritura como herramientas de aprendizaje de los contenidos de sus materias. Ello, sin embargo, no puede de ninguna manera interpretarse como el resultado de algún tipo de heroísmo o voluntarismo ciego por parte de los educadores; como sostiene Paula Carlino, para que los docentes se sientan capaces y puedan hacerse cargo de enseñar a leer y a escribir en sus cátedras, es imprescindible que las instituciones, a partir de políticas educativas y curriculares, respalden y reconozcan la necesidad de transformar las prácticas de enseñanza a partir de acciones y recursos concretos que se sostengan en el tiempo.
Así y todo, el libro "Escribir, leer y aprender: una introducción a la alfabetización académica" nos convence de que no hay impedimentos insalvables para que cada educador, sin mayores dilaciones, se ponga manos a la obra para buscar mejorar su enseñanza y la calidad de los aprendizajes de sus alumnos a través de ocuparse de la lectura y la escritura en el aula universitaria.

\section{Referencias}

Altrichter, H., Kemmis, S., McTaggart, R., \& ZuberSkerrit, O. (2002). The concept of action research. The learning organization, 9(3), 125-131.

Carlino, P., \& Martínez, S. (Eds.). (2009). La lectura y la escritura: un asunto de todos. Neuquén: Universidad Nacional del Comahue.

Langer, J. A., \& Applebee, A. N. (1987). How Writing Shapes Thinking: A Study of Teaching and Learning. Research Report No. 22. Urbana, Illinois: National Council of Teachers of English.

Wells, G. (1990a). Creating the Conditions to Encourage Literate Thinking. Educational Leadership, 47(6), 13-17.

Wells, G. (1990b). Talk about Text: Where Literacy Is Learned and Taught. Curriculum Inquiry, 20(4), 369405.

${ }^{1}$ Carlino, P. (2005). Escribir, leer y aprender en la universidad: una introducción a la alfabetización académica. Buenos Aires: Fondo de Cultura Económica de Argentina.

2 Paula Carlino : es Doctora en Psicología de la Educación por la Universidad Autónoma de Madrid y, actualmente, se desempeña como Investigadora Independiente del Consejo Nacional de Investigaciones Científicas y Técnicas-CONICET-, Argentina, con sede de trabajo en el Instituto de Lingüística de la Facultad de Filosofía y Letras de la Universidad de Buenos Aires. Desde el año 2006, dirige el Grupo para la Inclusión y Calidad Educativas a través de Ocuparnos de la Lectura y la Escritura en todas las Materias (GICEOLEM). 